\title{
Asynchronous Iterative Waterfilling for Gaussian Frequency-Selective Interference Channels
}

\author{
Gesualdo Scutari ${ }^{1}$, Daniel P. Palomar ${ }^{2}$, and Sergio Barbarossa ${ }^{1}$ \\ E-mail: \{scutari, sergio\}@infocom.uniroma1.it, palomar@ust.hk \\ ${ }^{1}$ Dpt. INFOCOM, Univ. of Rome "La Sapienza", Via Eudossiana 18, 00184 Rome, Italy. \\ ${ }^{2}$ Dpt. of Electronic and Computer Eng., Hong Kong Univ. of Science and Technology, Hong Kong. \\ Submitted to IEEE Transactions on Information Theory, August 22, 2006. \\ Revised September 25, 2007. Accepted January 14, 2008.*
}

\begin{abstract}
This paper considers the maximization of information rates for the Gaussian frequency-selective interference channel, subject to power and spectral mask constraints on each link. To derive decentralized solutions that do not require any cooperation among the users, the optimization problem is formulated as a static noncooperative game of complete information. To achieve the so-called Nash equilibria of the game, we propose a new distributed algorithm called asynchronous iterative waterfilling algorithm. In this algorithm, the users update their power spectral density in a completely distributed and asynchronous way: some users may update their power allocation more frequently than others and they may even use outdated measurements of the received interference. The proposed algorithm represents a unified framework that encompasses and generalizes all known iterative waterfilling algorithms, e.g., sequential and simultaneous versions. The main result of the paper consists of a unified set of conditions that guarantee the global converge of the proposed algorithm to the (unique) Nash equilibrium of the game.
\end{abstract}

Index Terms: Game theory, Gaussian frequency-selective interference channel, Nash equilibrium, totally asynchronous algorithm, iterative waterfilling algorithm.

\section{Introduction and Motivation}

In this paper we focus on the frequency selective interference channel with Gaussian noise. The capacity region of the interference channel is still unknown. Only some bounds are available (see, e.g., [1, 2, for a summary of the known results about the Gaussian interference channel). A pragmatic approach that leads to an achievable region or inner bound of the capacity region is to restrict the system to operate as a set of independent units, i.e., not allowing multiuser encoding/decoding or the use of interference cancelation techniques. This achievable region is very relevant in practical systems with limitations on

*Part of this work was presented in IEEE Workshop on Signal Processing Advances in Wireless Communications, (SPAWC-2006), July 2-5, 2006, and in Information Theory and Applications (ITA) Workshop, Jan. 29 - Feb. $2,2007$. This work was supported by the SURFACE project funded by the European Community under Contract IST-4-027187STP-SURFACE. 
the decoder complexity and simplicity of the system. With this assumption, multiuser interference is treated as noise and the transmission strategy for each user is simply his power allocation. The system design reduces then to finding the optimum Power Spectral Density (PSD) for each user, according to a specified performance metric.

Within this context, existing works [4]-[15] considered the maximization of the information rates of all the links, subject to transmit power and (possibly) spectral mask constraints on each link. The latter constraints are especially motivated in adaptive scenarios, e.g., cognitive radio, where previously allocated spectral bands may be reused, but provided that the generated interference falls below specified thresholds [3]. In [4, 5], a centralized approach based on duality theory was proposed to compute, under technical conditions, the largest achievable rate region of the system (i.e., the Pareto-optimal set of the achievable rates). Our interest, in this paper, is focused on finding distributed algorithms with no centralized control and no cooperation among the users. Hence, we cast the system design under the convenient framework of game theory. In particular, we formulate the rate maximization problem as a strategic non-cooperative game of complete information, where every link is a player that competes against the others by choosing the spectral power allocation that maximizes his own information rate. An equilibrium for the whole system is reached when every player is unilaterally optimum, i.e., when, given the current strategies of the others, any change in his own strategy would result in a rate loss. This equilibrium constitutes the celebrated notion of Nash Equilibrium (NE) [17].

The Nash equilibria of the rate maximization game can be reached using Gaussian signaling and a proper PSD from each user [9]-[12]. To obtain the optimal PSD of the users, Yu, Ginis, and Cioffi proposed the sequential Iterative WaterFilling Algorithm (IWFA) [6] in the context of DSL systems, modeled as a Gaussian frequency-selective interference channel. The algorithm is an instance of the Gauss-Seidel scheme [18]: the users maximize their own information rates sequentially (one after the other), according to a fixed updating order. Each user performs the single-user waterfilling solution given the interference generated by the others as additive (colored) noise. The most appealing features of the sequential IWFA are its low-complexity and distributed nature. In fact, to compute the waterfilling solution, each user only needs to measure the noise-plus-interference PSD, without requiring specific knowledge of the power allocations and the channel transfer functions of all other users. The convergence of the sequential IWFA has been studied in a number of works [7], 11]-[15], each time obtaining milder convergence conditions. However, despite its appealing properties, the sequential IWFA may suffer from slow convergence if the number of users in the network is large, just because of the sequential updating strategy. In addition, the algorithm requires some form of central scheduling to determine the order in which users update their PSD.

To overcome the drawback of slow speed of convergence, the simultaneous IWFA was proposed in [9, 11, 12]. The simultaneous IWFA is an instance of the Jacobi scheme [18]: at each iteration, the users update their own strategies simultaneously, still according to the waterfilling solution, but using the interference generated by the others in the previous iteration. The simultaneous IWFA was shown to 
converge to the unique NE of the rate maximization game faster than the sequential IWFA and under weaker conditions on the multiuser interference than those given in [6, 7], 13]- 15] for the sequential IWFA. Furthermore, differently from [6, 17, 13, 15, the algorithm as proposed in [1] takes explicitly into account the spectral masks constraints. However, the simultaneous IWFA still requires some form of synchronization, as all the users need to be updated simultaneously. Clearly, in a real network with many users, the synchronization requirement of both sequential and simultaneous IWFAs goes against the non-cooperation principle and it might be unacceptable.

This paper generalizes the existing results for the sequential and simultaneous IWFAs and develops a unified framework based on the so-called asynchronous IWFA, that falls within the class of totally asynchronous schemes of [18]. In this more general algorithm, all users still update their power allocations according to the waterfilling solution, but the updates can be performed in a totally asynchronous way (in the sense of [18]). This means that some users may update their power allocations more frequently than others and they may even use an outdated measurement of the interference caused from the others. These features make the asynchronous IWFA appealing for all practical scenarios, either wired or wireless, as it strongly relaxes the need for coordinating the users' updating schedule.

The main contribution of this paper is to derive sufficient conditions for the global convergence of the asynchronous IWFA to the (unique) NE of the rate maximization game. Interestingly, our convergence conditions are shown to be independent of the users' update schedule. Hence, they represent a unified set of conditions encompassing all existing algorithms, either synchronous or asynchronous, that can be seen as special cases of our asynchronous IWFA. Our conditions also imply that both sequential and simultaneous algorithms are robust to situations where some users may fail to follow their updating schedule. Finally, we show that our sufficient conditions for the convergence of the asynchronous IWFA coincide with those given recently in [11] for the convergence of the (synchronous) sequential and simultaneous IWFAs, and are larger than conditions obtained in [6, 7], [13] - 15] for the convergence of the sequential IWFA in the absence of spectral mask constraints.

The paper is organized as follows. Section 2 provides the system model and formulates the optimization problem as a strategic non-cooperative game. Section 3 contains the main result of the paper: the description of the proposed asynchronous IWFA along with its convergence properties. Section 4 recovers the sequential and simultaneous IWFAs as special cases of the asynchronous IWFA and then, as a by product, it provides a unified set of convergence conditions for both algorithms. Finally, Section 5 draws some conclusions.

\section{System Model and Problem Formulation}

In this section we clarify the assumptions and the constraints underlying the system model and we formulate the optimization problem addressed in this paper explicitly. 


\subsection{System model}

We consider a Gaussian frequency-selective interference channel composed by multiple links. Since our goal is to find distributed algorithms that require neither a centralized control nor a coordination among the users, we focus on transmission techniques where interference cancelation is not possible and multiuser interference is treated by each receiver as additive colored noise. The channel frequencyselectivity is handled, with no loss of optimality, adopting a multicarrier transmission strategy 1 Given the above system model, we make the following assumptions:

A.1 Each channel changes sufficiently slowly to be considered fixed during the whole transmission, so that the information theoretic results are meaningful;

A.2 The channel from each source to its own destination is known to the intended receiver, but not to the other terminals; each receiver is also assumed to measure with no errors the overall PSD of the noise plus interferences generated by the other users. Based on this information, each receiver computes the optimal power allocation across the frequency bins for its own transmitter and feeds it back to its transmitter through a low bit rate (error-free) feedback channel 2

A.3 All the users are block-synchronized with an uncertainty at most equal to the cyclic prefix length. This imposes a minimum length of the cyclic prefix that will depend on the maximum channel delay spread.

We consider the following constraints:

Co.1 Maximum overall transmit power for each user:

$$
E\left\{\left\|\mathbf{s}_{q}\right\|_{2}^{2}\right\}=\sum_{k=1}^{N} \bar{p}_{q}(k) \leq N P_{q}, \quad q=1, \ldots, Q,
$$

where $\mathbf{s}_{q}$ contains the $N$ symbols transmitted by user $q$ on the $N$ carriers, $\bar{p}_{q}(k) \triangleq E\left\{\left|s_{q}(k)\right|^{2}\right\}$ denotes the power allocated by user $q$ over carrier $k$, and $P_{q}$ is power in units of energy per transmitted symbol. Co.2 Spectral mask constraints:

$$
E\left\{\left|s_{q}(k)\right|^{2}\right\}=\bar{p}_{q}(k) \leq \bar{p}_{q}^{\max }(k), \quad k=1, \ldots, N, \quad q=1, \ldots, Q
$$

where $\bar{p}_{q}^{\max }(k)$ represents the maximum power that is allowed to be allocated on the $k$-th frequency bin from the $q$-th user. Constraints like (2) are imposed to limit the amount of interference generated by each transmitter over pre-specified bands.

The main goal of this paper is to obtain the optimal vector power allocation $\mathbf{p}_{q} \triangleq\left(p_{q}(1), \ldots, p_{q}(N)\right)$, for each user, according to the optimality criterion introduced in the next section.

\footnotetext{
${ }^{1}$ Multicarrier transmission is a capacity-lossless strategy for sufficiently large block length [21, 22].

${ }^{2}$ In practice, both measurements and feedback are inevitably affected by errors. This scenario can be studied by extending our formulation to games with partial information [23, 24], but this goes beyond the scope of the present paper.
} 


\subsection{Problem formulation as a game}

We consider a strategic non-cooperative game [23, 24, in which the players are the links and the payoff functions are the information rates on each link: Each player competes rationally 3 against the others by choosing the strategy that maximizes his own rate, given constraints Co.1 and Co.2. A NE of the game is reached when every user, given the strategy profile of the others, does not get any rate increase by changing his own strategy.

Using the signal model described in Section 2.1, the achievable rate for each player $q$ is computed as the maximum information rate on the $q$-th link, assuming all the other received signals as additive colored noise. It is straightforward to see that a (pure or mixed strategy) NE is obtained if each user transmits using Gaussian signaling, with a proper PSD. In fact, for each user, given that all other users use Gaussian codebooks, the optimal codebook maximizing mutual information is also Gaussian [21] 4 Hence, the maximum achievable rate for the $q$-th user is given by [21]

$$
R_{q}=\frac{1}{N} \sum_{k=1}^{N} \log \left(1+\operatorname{sinr}_{q}(k)\right)
$$

with $\operatorname{sinr}_{q}(k)$ denoting the Signal-to-Interference plus Noise Ratio (SINR) on the $k$-th carrier for the $q$-th link:

$$
\operatorname{sinr}_{q}(k) \triangleq \frac{\left|\bar{H}_{q q}(k)\right|^{2} \bar{p}_{q}(k) / d_{q q}^{\gamma}}{\sigma_{q}^{2}(k)+\sum_{r \neq q}\left|\bar{H}_{q r}(k)\right|^{2} \bar{p}_{r}(k) / d_{r q}^{\gamma}} \triangleq \frac{\left|H_{q q}(k)\right|^{2} p_{q}(k)}{\sigma_{q}^{2}(k)+\sum_{r \neq q}\left|H_{q r}(k)\right|^{2} p_{r}(k)},
$$

where $\bar{H}_{q r}(k)$ denotes the frequency-response of the channel between source $r$ and destination $q$ excluding the path-loss $d_{q r}^{\gamma}$ with exponent $\gamma$ and $d_{q r}$ is the distance between source $r$ and destination $q ; \sigma_{q}^{2}(k)$ is the variance of the zero mean circularly symmetric complex Gaussian noise at receiver $q$ over the carrier $k$; and for the convenience of notation, we have introduced the normalized quantities $H_{q r}(k) \triangleq \bar{H}_{q r}(k) \sqrt{P_{r} / d_{q r}^{\gamma}}$ and $p_{q}(k) \triangleq \bar{p}_{q}(k) / P_{q}$.

Observe that in the case of practical coding schemes, where only finite order constellations can be used, we can use the gap approximation analysis [25, 26] and write the number of bits transmitted over the $N$ substreams from the $q$-th source still as in (3) but replacing $\left|H_{q q}(k)\right|^{2}$ in (44) with $\left|H_{q q}(k)\right|^{2} / \Gamma_{q}$, where $\Gamma_{q} \geq 1$ is the gap. The gap depends only on the family of constellation and on $P_{e, q}$; for $M$-QAM constellations, for example, if the symbol error probability is approximated by $P_{e, q}\left(\operatorname{sinr}_{q}(k)\right) \approx 4 \mathcal{Q}\left(\sqrt{3 \operatorname{sinr}_{q}(k) /(M-1)}\right)$, the resulting gap is $\Gamma_{q}=\left(\mathcal{Q}^{-1}\left(P_{e, q} / 4\right)\right)^{2} / 3$ [25, 26].

In summary, we have a game with the following structure:

$$
\mathscr{G}=\left\{\Omega,\left\{\mathscr{P}_{q}\right\}_{q \in \Omega},\left\{R_{q}\right\}_{q \in \Omega}\right\}
$$

\footnotetext{
${ }^{3}$ The rationality assumption means that each user will never chose a strictly dominated strategy. A strategy profile $\mathbf{x}_{q}$ is strictly dominated by $\mathbf{z}_{q}$ if $\Phi_{q}\left(\mathbf{x}_{q}, \mathbf{y}_{-q}\right)<\Phi_{q}\left(\mathbf{z}_{q}, \mathbf{y}_{-q}\right)$, for a given admissible $\mathbf{y}_{-q} \triangleq\left(\mathbf{y}_{1}, \ldots, \mathbf{y}_{q-1}, \mathbf{y}_{q+1}, \ldots, \mathbf{y}_{Q}\right)$, where $\Phi_{q}$ denotes the payoff function of player $q$.

${ }^{4}$ Observe that, in general, Nash equilibria achievable using arbitrary non-Gaussian codes may exist. In this paper, we focus only on transmission using Gaussian codebooks.
} 
where $\Omega \triangleq\{1,2, \ldots, Q\}$ denotes the set of the $Q$ active links, $\mathscr{P}_{q}$ is the set of admissible (normalized) power allocation strategies, across the $N$ available carriers, for the $q$-th player, defined a 5

$$
\mathscr{P}_{q} \triangleq\left\{\mathbf{p}_{q} \in \mathbb{R}^{N}: \frac{1}{N} \sum_{k=1}^{N} p_{q}(k)=1, \quad 0 \leq p_{q}(k) \leq p_{q}^{\max }(k), \quad k=1, \ldots, N\right\},
$$

with $p_{q}^{\max }(k) \triangleq \bar{p}_{q}^{\max }(k) / P_{q}$ and $R_{q}$ is the payoff function of the $q$-th player, defined in (3).

The optimal strategy for the $q$-th player, given the power allocation of the others, is then the solution to the following maximization problem 6

$$
\begin{array}{ll}
\underset{\mathbf{p}_{q}}{\operatorname{maximize}} & \frac{1}{N} \sum_{k=1}^{N} \log \left(1+\operatorname{sinr}_{q}(k)\right), \\
\text { subject to } & \mathbf{p}_{q} \in \mathscr{P}_{q}
\end{array}
$$

where $\operatorname{sinr}_{q}(k)$ and $\mathscr{P}_{q}$ and are given in (4) and (6) , respectively. Note that, for each $q$, the maximum in (7) is taken over $\mathbf{p}_{q}$, for a fixed $\mathbf{p}_{-q} \triangleq\left(\mathbf{p}_{1}, \ldots, \mathbf{p}_{q-1}, \mathbf{p}_{q+1}, \ldots, \mathbf{p}_{Q}\right)$.

The solutions of (7) are the well-known Nash Equilibria, which are formally defined as follows.

Definition $1 A$ (pure) strategy profile $\mathbf{p}^{\star}=\left(\mathbf{p}_{1}^{*}, \ldots, \mathbf{p}_{Q}^{*}\right) \in \mathscr{P}_{1} \times \ldots \times \mathscr{P}_{Q}$ is a Nash Equilibrium of the game $\mathscr{G}$ in (5) if

$$
R_{q}\left(\mathbf{p}_{q}^{\star}, \mathbf{p}_{-q}^{\star}\right) \geq R_{q}\left(\mathbf{p}_{q}, \mathbf{p}_{-q}^{\star}\right), \quad \forall \mathbf{p}_{q} \in \mathscr{P}_{q}, \forall q \in \Omega .
$$

Observe that, for the payoff functions defined in (3), we can indeed limit ourselves to adopt pure strategies w.l.o.g., as we did in (5), since every $\mathrm{NE}$ of the game is proved to be achievable using pure strategies in [10].

According to (7), all the (pure) Nash equilibria of the game, if they exist, must satisfy the waterfilling solution for each user, i.e., the following system of nonlinear equations:

$$
\mathbf{p}_{q}^{\star}=\mathrm{WF}_{q}\left(\mathbf{p}_{1}^{\star}, \ldots, \mathbf{p}_{q-1}^{\star}, \mathbf{p}_{q+1}^{\star}, \ldots, \mathbf{p}_{Q}^{\star}\right)=\mathrm{WF}_{q}\left(\mathbf{p}_{-q}^{\star}\right), \quad \forall q \in \Omega,
$$

with the waterfilling operator $\mathrm{WF}_{q}(\cdot)$ defined as

$$
\left[\mathrm{WF}_{q}\left(\mathbf{p}_{-q}\right)\right]_{k} \triangleq\left[\mu_{q}-\frac{\sigma_{q}^{2}(k)+\sum_{r \neq q}\left|H_{q r}(k)\right|^{2} p_{r}(k)}{\left|H_{q q}(k)\right|^{2}}\right]_{0}^{p_{q}^{\max }(k)}, \quad k=1, \ldots, N,
$$

where $[x]_{a}^{b}$ denotes the Euclidean projection of $x$ onto the interval $[a, b]$.7 The water-level $\mu_{q}$ is chosen to satisfy the power constraint $(1 / N) \sum_{k=1}^{N} p_{q}^{\star}(k)=1$.

\footnotetext{
${ }^{5}$ In order to avoid the trivial solution $p_{q}^{\star}(k)=p_{q}^{\max }(k)$ for all $k, \sum_{k=1}^{N} p_{q}^{\max }(k)>N$ is assumed for all $q \in \Omega$. Furthermore, in the feasible strategy set of each player, we can replace, without loss of generality, the original inequality power constraint $(1 / N) \sum_{k=1}^{N} p_{q}(k) \leq 1$, with equality, since, at the optimum, this constraint must be satisfied with equality.

${ }^{6}$ In the optimization problem in (7), any concave strictly increasing function of the rate can be equivalently considered as payoff function of each player. The optimal solutions of this new set of problems coincide with those of (7).

${ }^{7}$ The Euclidean projection $[x]_{a}^{b}$, with $a \leq b$, is defined as follows: $[x]_{a}^{b}=a$, if $x \leq a,[x]_{a}^{b}=x$, if $a<x<b$, and $[x]_{a}^{b}=b$, if $x \geq b$.
} 
Observe that in the absence of spectral mask constraints (i.e., when $p_{q}^{\max }(k)=+\infty, \forall q, \forall k$ ), the Nash equilibria of game $\mathscr{G}$ are given by the classical simultaneous waterfilling solutions [6, 7], where $\mathrm{WF}_{q}(\cdot)$ in (9) is still obtained from (10) simply setting $p_{q}^{\max }(k)=+\infty, \forall q, \forall k$. Interestingly, the presence of spectral mask constraints does not affect the existence of a pure-strategies NE of game $\mathscr{G}$, as stated in the following.

Proposition 1 The game $\mathscr{G}$ in (5) always admits at least one pure-strategy NE, for any set of channel realizations, power and spectral mask constraints.

Proof. The proof comes from standard results of game theory [23, 24] and it is given in [10].

In general, the game $\mathscr{G}$ may admit multiple equilibria, depending on the level of multiuser interference [10]. In the forthcoming sections, we provide sufficient conditions ensuring the uniqueness of the $\mathrm{NE}$ and we address the problem of how to reach such an equilibrium in a totally distributed way.

\section{Asynchronous Iterative Waterfilling}

To reach the NE of game $\mathscr{G}$, we propose a totally asynchronous distributed iterative waterfilling procedure, which we name asynchronous Iterative WaterFilling Algorithm. The proposed algorithm can be seen as an instance of the totally asynchronous scheme of [18]: all the users maximize their own rate in a totally asynchronous way. More specifically, some users are allowed to update their strategy more frequently than the others, and they might perform their updates using outdated information about the interference caused from the others. What we show is that the asynchronous IWFA converges to a stable NE of game $\mathscr{G}$, whichever the updating schedule is, under rather mild conditions on the multiuser interference. Interestingly, these conditions are also sufficient to guarantee the uniqueness of the NE.

To provide a formal description of the asynchronous IWFA, we need to introduce some preliminary definitions. We assume, without any loss of generality, that the set of times at which one or more users update their strategies is the discrete set $\mathcal{T}=\mathbb{N}_{+}=\{0,1,2, \ldots\}$. Let $\mathbf{p}_{q}^{(n)}$ denote the vector power allocation of user $q$ at the $n$-th iteration, and let $\mathcal{T}_{q} \subseteq \mathcal{T}$ denote the set of times $n$ at which user $q$ updates his power vector $\mathbf{p}_{q}^{(n)}$ (thus, implying that, at time $n \notin \mathcal{T}_{q}, \mathbf{p}_{q}^{(n)}$ is left unchanged). Let $\tau_{r}^{q}(n)$ denote the most recent time at which the interference from user $r$ is perceived by user $q$ at the $n$-th iteration (observe that $\tau_{r}^{q}(n)$ satisfies $0 \leq \tau_{r}^{q}(n) \leq n$ ). Hence, if user $q$ updates its power allocation at the $n$-th iteration, then it waterfills, according to (10), the interference level caused by the power allocations of the others:

$$
\mathbf{p}_{-q}^{\left(\tau^{q}(n)\right)} \triangleq\left(\mathbf{p}_{1}^{\left(\tau_{1}^{q}(n)\right)}, \ldots, \mathbf{p}_{q-1}^{\left(\tau_{q-1}^{q}(n)\right)}, \mathbf{p}_{q+1}^{\left(\tau_{q+1}^{q}(n)\right)}, \ldots, \mathbf{p}_{Q}^{\left(\tau_{Q}^{q}(n)\right)}\right)
$$

The overall system is said to be totally asynchronous if the following weak assumptions are satisfied for each $q$ [18]: A1) $0 \leq \tau_{r}^{q}(n) \leq n$; A2) $\lim _{k \rightarrow \infty} \tau_{r}^{q}\left(n_{k}\right)=+\infty$; and A3) $\left|\mathcal{T}_{q}\right|=\infty$; where $\left\{n_{k}\right\}$ is a sequence of elements in $\mathcal{T}_{q}$ that tends to infinity. Assumptions A1-A3 are standard in asynchronous convergence theory [18, and they are fulfilled in any practical implementation. In fact, A1 simply 
indicates that, at any given iteration $n$, each user $q$ can use only the interference vectors $\mathbf{p}_{-q}^{\left(\tau^{q}(n)\right)}$ allocated by the other users in the previous iterations (to preserve causality). Assumption A2 states that, for any given iteration index $n_{k}$, the values of the components of $\mathbf{p}_{-q}^{\left(\tau^{q}(n)\right)}$ in (11) generated prior to $n_{k}$, are not used in the updates of $\mathbf{p}_{q}^{(n)}$, when $n$ becomes sufficiently larger than $n_{k}$; which guarantees that old information is eventually purged from the system. Finally, assumption A3 indicates that no user fails to update its own strategy as time $n$ goes on.

Given game $\mathscr{G}$, let $\mathcal{D}_{q}^{\text {min }} \subseteq\{1, \cdots, N\}$ denote the set $\{1, \ldots, N\}$ (possibly) deprived of the carrier indices that user $q$ would never use as the best response set to any strategies adopted by the other users [10]:

$$
\mathcal{D}_{q}^{\min } \triangleq\left\{k \in\{1, \ldots, N\}: \exists \mathbf{p}_{-q} \in \mathscr{P}_{-q} \text { such that }\left[\mathrm{WF}_{q}\left(\mathbf{p}_{-q}\right)\right]_{k} \neq 0\right\},
$$

with $\mathrm{WF}_{q}(\cdot)$ defined in (10) and $\mathscr{P}_{-q} \triangleq \mathscr{P}_{1} \times \cdots \times \mathscr{P}_{q-1} \times \mathscr{P}_{q+1} \times \cdots \times \mathscr{P}_{Q}$. In [10], an iterative procedure to obtain a set $\mathcal{D}_{q}$ such that $\mathcal{D}_{q}^{\text {min }} \subseteq \mathcal{D}_{q} \subseteq\{1, \cdots, N\}$ is given. Let the matrix $\mathbf{S}^{\text {max }} \in \mathbb{R}_{+}^{Q \times Q}$ be defined as

$$
\left[\mathbf{S}^{\max }\right]_{q r} \triangleq \begin{cases}\max _{k \in \mathcal{D}_{q} \cap \mathcal{D}_{r}} \frac{\left|\bar{H}_{q r}(k)\right|^{2}}{\left|\bar{H}_{q q}(k)\right|^{2}} \frac{d_{q q}^{\gamma}}{d_{q r}^{\gamma}} \frac{P_{r}}{P_{q}}, & \text { if } r \neq q \\ 0, & \text { otherwise }\end{cases}
$$

with the convention that the maximum in (13) is zero if $\mathcal{D}_{q} \cap \mathcal{D}_{r}$ is empty. In (13), each set $\mathcal{D}_{q}$ can be chosen as any subset of $\{1, \cdots, N\}$ such that $\mathcal{D}_{q}^{\text {min }} \subseteq \mathcal{D}_{q} \subseteq\{1, \cdots, N\}$, with $\mathcal{D}_{q}^{\text {min }}$ defined in (12). Using the above notation, the asynchronous IWFA is described in Algorithm 1 (where $\mathrm{N}_{\mathrm{it}}$ denotes the number of iterations).

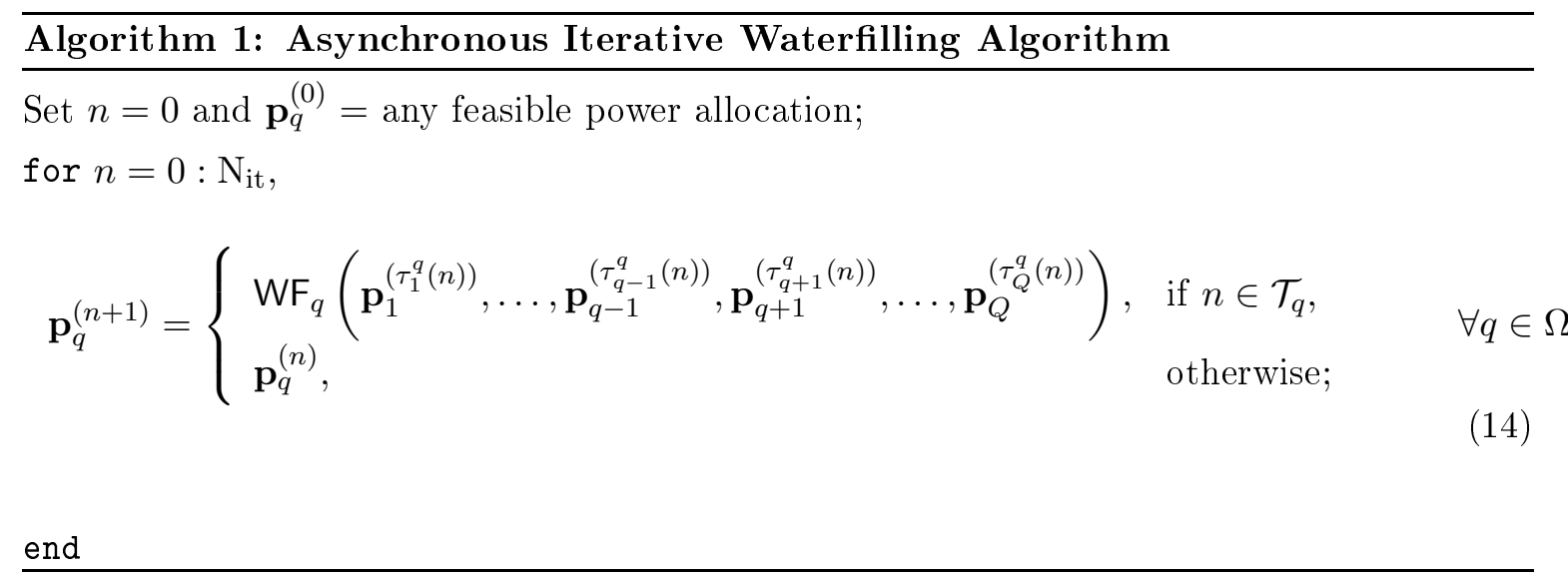

The convergence of the algorithm is guaranteed under the following sufficient conditions.

Theorem 1 Assume that the following condition is satisfied:

$$
\rho\left(\mathbf{S}^{\max }\right)<1
$$

where $\mathbf{S}^{\max }$ is defined in (13) and $\rho\left(\mathbf{S}^{\mathrm{max}}\right)$ denotes the spectral radiud 8 of $\mathbf{S}^{\mathrm{max}}$. Then, as $\mathrm{N}_{\mathrm{it}} \rightarrow \infty$,

\footnotetext{
${ }^{8}$ The spectral radius $\rho(\mathbf{S})$ of the matrix $\mathbf{S}$ is defined as $\rho(\mathbf{S})=\max \{|\lambda|: \lambda \in \operatorname{eig}(\mathbf{S})\}$, with eig $(\mathbf{S})$ denoting the set of eigenvalues of $\mathbf{S}[27]$.
} 
the asynchronous IWFA described in Algorithm 1 converges to the unique NE of game $\mathscr{G}$, for any set of feasible initial conditions and updating schedule.

Proof. The proof consists in showing that, under (C1), conditions of the Asynchronous Convergence Theorem in [18] are satisfied. A key point in the proof is given by the following property of the multiuser waterfilling mapping $\mathrm{WF}(\mathbf{p})=\left(\mathrm{WF}_{q}\left(\mathbf{p}_{-q}\right)\right)_{q \in \Omega}$ based on the interpretation of the waterfilling solution (10) as a proper projector [11]:

$$
\left\|\mathrm{WF}\left(\mathbf{p}^{(1)}\right)-\mathrm{WF}\left(\mathbf{p}^{(2)}\right)\right\| \leq \beta\left\|\mathbf{p}^{(1)}-\mathbf{p}^{(2)}\right\|, \quad \forall \mathbf{p}^{(1)}, \mathbf{p}^{(2)} \in \mathscr{P},
$$

where $\|\cdot\|$ is a proper vector norm and $\beta$ is a positive constant, which is less than 1 if condition (C1) is satisfied. See Appendix $\mathrm{A}$ for the details.

To give additional insight into the physical interpretation of the convergence conditions of Algorithm 1, we provide the following corollary of Theorem 1 .

Corollary 1 A sufficient condition for (C1) in Theorem 1 is given by one of the two following set of conditions:

$$
\begin{aligned}
& \frac{1}{w_{q}} \sum_{r \neq q} \max _{k \in \mathcal{D}_{r} \cap \mathcal{D}_{q}}\left\{\frac{\left|\bar{H}_{q r}(k)\right|^{2}}{\left|\bar{H}_{q q}(k)\right|^{2}}\right\} \frac{d_{q q}^{\gamma}}{d_{q r}^{\gamma}} \frac{P_{r}}{P_{q}} w_{r}<1, \quad \forall q \in \Omega, \\
& \frac{1}{w_{r}} \sum_{q \neq r} \max _{k \in \mathcal{D}_{r} \cap \mathcal{D}_{q}}\left\{\frac{\left|\bar{H}_{q r}(k)\right|^{2}}{\left|\bar{H}_{q q}(k)\right|^{2}}\right\} \frac{d_{q q}^{\gamma}}{d_{q r}^{\gamma}} \frac{P_{r}}{P_{q}} w_{q}<1, \quad \forall r \in \Omega,
\end{aligned}
$$

where $\mathbf{w} \triangleq\left[w_{1}, \ldots, w_{Q}\right]^{T}$ is any positive vector 9

Note that, according to the definition of $\mathcal{D}_{q}$ in (13), one can always choose $\mathcal{D}_{q}=\{1, \ldots, N\}$ in (C1) and (C2)-(C3). However, less stringent conditions are obtained by removing unnecessary carriers, i.e., the carriers that, for the given power budget and interference levels, are never going to be used.

Recall that, if finite order constellations are used, Theorem 1 is still valid using the gap-approximation method [25, 26] as pointed out in Section 2.2, It is sufficient to replace each $\left|H_{q q}(k)\right|^{2}$ in (C1) with $\left|H_{q q}(k)\right|^{2} / \Gamma_{q}$.

Remark 1 - Global convergence and robustness of the algorithm: Even though the rate maximization game in (7) and the consequent waterfilling mapping (10) are nonlinear, condition (C1) guarantees the global convergence of the asynchronous IWFA. Observe that Algorithm 1 contains as special cases a plethora of algorithms, each one obtained by a possible choice of the scheduling of the users in the updating procedure (i.e., the parameters $\left\{\tau_{r}^{q}(n)\right\}$ and $\left\{\mathcal{T}_{q}\right\}$ ). The important result stated in Theorem 1 is that all the algorithms resulting as special cases of the asynchronous IWFA are guaranteed to reach the unique NE of the game, under the same set of convergence conditions (provided

\footnotetext{
${ }^{9}$ The optimal positive vector $\mathbf{w}$ in $(\underline{\mathrm{C} 2})-(\underline{\mathrm{C} 3})$ is given by the solution of a geometric programming, as shown in [11 Corollary 5]
} 
that A1-A3 are satisfied), since condition (C1) does not depend on the particular choice of $\left\{\mathcal{T}_{q}\right\}$ and $\left\{\tau_{r}^{q}(n)\right\}$.

Remark 2 - Physical interpretation of convergence conditions: As expected, the convergence of the asynchronous IWFA and the uniqueness of NE are ensured if the interferers are sufficiently far apart from the destinations. In fact, from ( $(\mathrm{C} 2)-(\mathrm{C} 3)$ one infers that, for any given set of channel realizations and power constraints, there exists a distance beyond which the convergence of the asynchronous IWFA (and the uniqueness of NE) is guaranteed, corresponding to the maximum level of interference that may be tolerated by each receiver [as quantified, e.g., in (르)] or that may be generated by each transmitter [as quantified, e.g., in (C3)]. But the most interesting result coming from (C1) and (C2)-(C3) is that the convergence of the asynchronous IWFA is robust against the worst normalized channels $\left|H_{q r}(k)\right|^{2} /$ $\left|H_{q q}(k)\right|^{2}$; in fact, the subchannels corresponding to the highest ratios $\left|H_{q r}(k)\right|^{2} /\left|H_{q q}(k)\right|^{2}$ (and, in particular, the subchannels where $\left|H_{q q}(k)\right|^{2}$ is vanishing) do not necessarily affect the convergence of the algorithm, as their carrier indices may not belong to the set $\mathcal{D}_{q}$.

Remark 3 - Distributed nature of the algorithm: Since the asynchronous IWFA is based on the waterfilling solution (10), it can be implemented in a distributed way, where each user, to maximize his own rate, only needs to measure the PSD of the overall interference-plus-noise and waterfill over it. More interestingly, according to the asynchronous scheme, the users may update their strategies using a potentially outdated version of the interference. Furthermore, some users are even allowed to update their power allocation more often than others, without affecting the convergence of the algorithm. These features strongly relax the constraints on the synchronization of the users' updates with respect to those imposed, for example, by the simultaneous or sequential updating schemes.

We can generalize the asynchronous IWFA given in Algorithm 1 by introducing a memory in the updating process, as given in Algorithm 2. We call this new algorithm smoothed asynchronous IWFA.

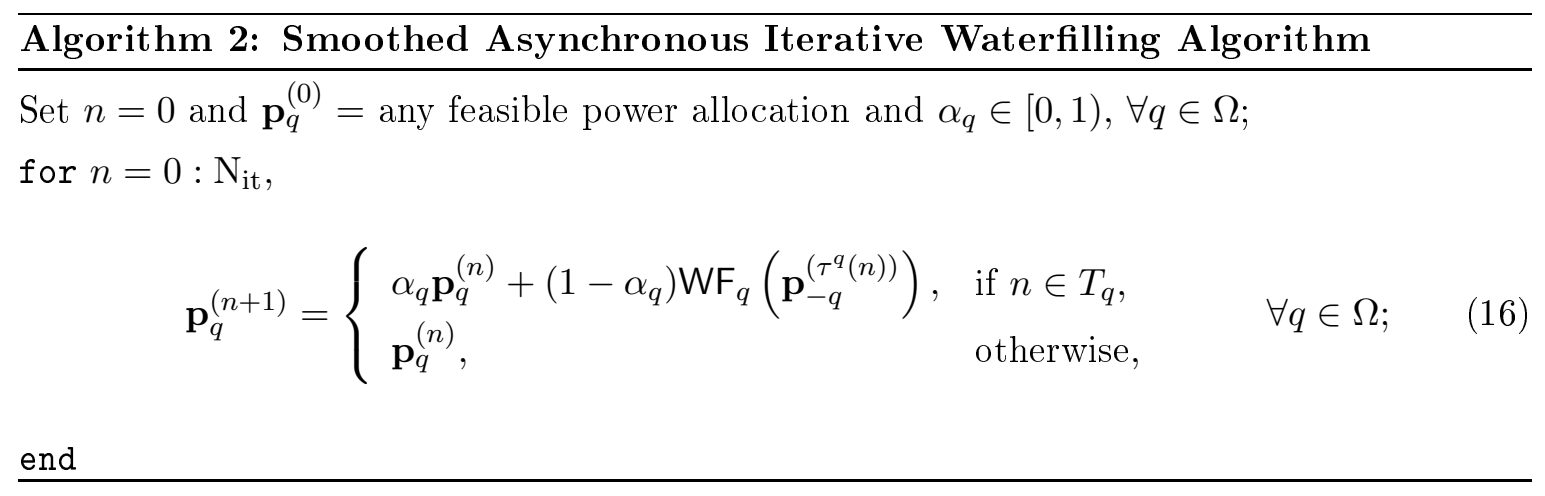

Each factor $\alpha_{q} \in[0,1)$ in Algorithm 2 can be interpreted as a forgetting factor: The larger $\alpha_{q}$ is, the longer the memory of the algorithm is 10 Interestingly the choice of $\alpha_{q}$ 's does not affect the

\footnotetext{
${ }^{10}$ In this paper, we are only considering constant channels. Nevertheless, in a time-varying scenario, (16) could be used to smooth the fluctuations due to the channel variations. In such a case, if the channel is fixed or highly stationary, it is convenient to take $\alpha_{q}$ close to 1 ; conversely, if the channel is rapidly varying, it is better to take a small $\alpha_{q}$.
} 
convergence capability of the algorithm (although it may affect the speed of convergence [11]), as proved in the following.

Theorem 2 Assume that condition of Theorem 1 is satisfied. Then, as $\mathrm{N}_{\mathrm{it}} \rightarrow \infty$, the smoothed asynchronous IWFA described in Algorithm 2 converges to the unique NE of game $\mathscr{G}$, for any set of feasible initial conditions, updating schedule, and $\left\{\alpha_{q}\right\}_{q \in \Omega}$, with $\alpha_{q} \in[0,1), \forall q \in \Omega$.

Proof. See Appendix A.

Remark 4 - Asynchronous IWFA in the presence of intercarrier interference: The proposed AIWFA can be extended to the case where the transmission by the different users contains time and frequency synchronization offsets. In [19, 20] we showed that the Asynchronous IWFA is robust against the intercarrier interference due to time and/or frequency offsets among the links and we derived sufficient conditions guaranteeing its convergence in the presence of such time/frequency misalignments.

\section{Two Special Cases: Sequential and Simultaneous IWFAs}

In this section, we specialize our asynchronous IWFA to two special cases: the sequential and the simultaneous IWFAs. As a by-product of the proposed unified framework, we show that both algorithms converge to the NE under the same sufficient conditions, that are larger than conditions obtained for the convergence of the sequential IWFA in [6, 7], [13, [15] (without considering the spectral mask constraints) and [14] (including the spectral mask constraints).

Sequential Iterative Waterfilling: The sequential IWFA is an instance of the Gauss-Seidel scheme by which each user is sequentially updated [18] based on the waterfilling mapping (10). In fact, in sequential IWFA each player, sequentially and according to a fixed order, maximizes his own rate (3), performing the single-user waterfilling solution in (10), given the others as interference. This scheme can also be seen as a particular case of the general asynchronous IWFA with the following parameters: $\mathcal{T}_{q}=\left\{k Q+q, \quad k \in \mathbb{N}_{+}\right\}=\{q, Q+q, 2 Q+q, \ldots\}$ and $\tau_{r}^{q}(n)=n, \forall r, q$. Using this settings in Algorithm 1, the sequential IWFA can be written in compact form as in Algorithm 3 ,

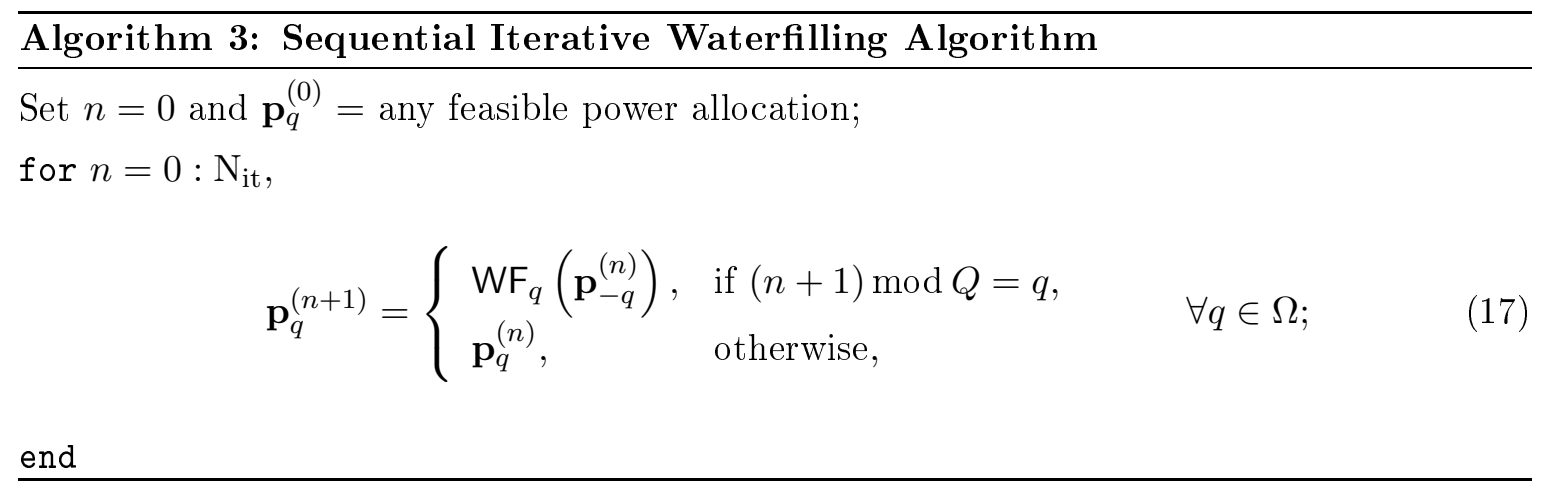


Simultaneous Iterative Waterfilling: The simultaneous IWFA can be interpreted as the synchronous Jacobi instance of the asynchronous IWFA. In fact, in the simultaneous IWFA, all the users update their own covariance matrix simultaneously at each iteration, performing the single user waterfilling solution (10), given the interference generated by the other users in the previous iteration. This is a particular case of the asynchronous IWFA in Algorithm 1 with the following parameters: $\mathcal{T}_{q}=\mathbb{N}_{+}$, and $\tau_{r}^{q}(n)=n, \forall r, q$, which leads to Algorithm 4 .

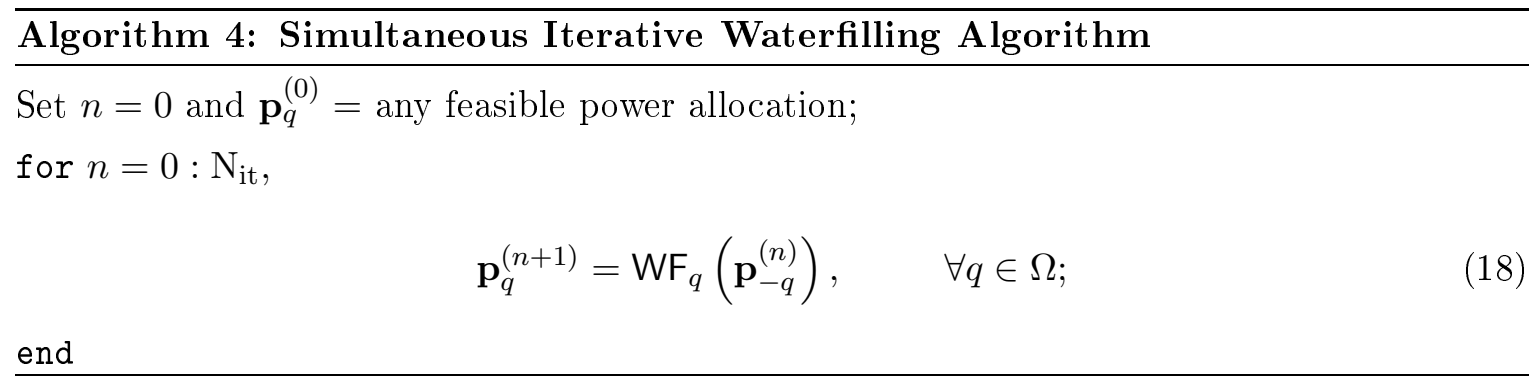

By direct product of our unified framework, invoking Theorem 1 we obtain the following unified set of convergence conditions for both sequential and simultaneous IWFAs [11].

Theorem 3 Assume that condition (C1) of Theorem 1 is satisfied. Then, as $\mathrm{N}_{\mathrm{it}} \rightarrow \infty$, the sequential and simultaneous IWFAs, described in Algorithm 3 and 4, respectively, converge geometrically to the unique NE of game $\mathscr{G}$ for any set of feasible initial conditions and updating schedule.

Remark 5 - Algorithm robustness: It follows form Theorem 3 that slight variations of the sequential or simultaneous IWFAs that fall within the unified framework of the asynchronous IWFA, are still guaranteed to converge, under the condition in Theorem 1. This means that, using for example Algorithm 3, if the order in the users' updates changes during time, or some user skips some update, or he uses an outdated version of the interference PSD, this does not affect the convergence of the algorithm. What is affected is only the convergence time. Moreover, as for the smoothed asynchronous IWFA, also for the sequential and simultaneous IWFAs described in Algorithm 3 and 4 , respectively, one can introduce a memory in the updating process [11, still guaranteeing convergence under conditions of Theorem 3 ,

Remark 6 - Comparison with previous convergence conditions: Algorithm 3 generalizes the well-known sequential iterative waterfilling algorithm proposed by Yu et al. in [6] to the case where the spectral mask constraints are explicitly taken into account. In fact, the algorithm in [6] can be obtained as a special case of Algorithm 3, by removing the spectral mask constraints in each set $\mathscr{P}_{q}$ in (6) ), (i.e. setting $p_{q}^{\max }(k)=+\infty, \forall k, q$ ), so that the waterfilling operator in (10) becomes the classical waterfilling solution [21, i.e., $\mathrm{WF}_{q}\left(\mathbf{p}_{-q}\right)=\left(\mu_{q} \mathbf{1}_{N}-\mathbf{i n s r}_{q}\right)^{+}$, where $(x)^{+}=\max (0, x)$ and $\operatorname{insr}_{q} \triangleq\left[\operatorname{insr}_{q}(1), \ldots, \operatorname{insr}_{q}(N)\right]^{T}$, with $\operatorname{insr}_{q}(k)=\left(\sigma_{w_{q}}^{2}(k)+\sum_{r \neq q}\left|H_{q r}(k)\right|^{2} p_{r}(k)\right) /\left|H_{q q}(k)\right|^{2}$. The convergence of the sequential IWFA has been studied in a number of works, either in the absence 
[6, 17, 8, 13, 15] or in the presence [11, 14] of spectral mask constraints. Interestingly, conditions in [6, 7, 8, 13, 15] and [14] imply our condition (두), which is more relaxed as shown next. Let

$$
\mathbf{\Upsilon} \triangleq\left(\mathbf{I}-\overline{\mathbf{S}}_{\mathrm{low}}^{\max }\right)^{-1} \overline{\mathbf{S}}_{\mathrm{upp}}^{\max }
$$

with $\overline{\mathbf{S}}_{\text {low }}^{\max }$ and $\overline{\mathbf{S}}_{\text {upp }}^{\max }$ denoting the strictly lower and strictly upper triangular part of the matrix $\overline{\mathbf{S}}^{\max }$, respectively, and $\overline{\mathbf{S}}^{\max }$ is defined similar to $\mathbf{S}^{\max }$ in (13), but taking the maximum over the whole set $\{1, \ldots, N\}$. The relationship between (sufficient) conditions for the convergence of sequential IWFA as derived in [6, 7, 8, 13, 14, 15] and condition (C1) 11 is given in the following corollary of Theorem 3 ,

Corollary 2 Sufficient conditions for (C1) in Theorem 1 are $[6]-[8] 12$

$$
\max _{k \in\{1, \ldots, N\}}\left\{\frac{\left|\bar{H}_{r q}(k)\right|^{2}}{\left|\bar{H}_{q q}(k)\right|^{2}}\right\} \frac{d_{q q}^{\alpha}}{d_{r q}^{\alpha}} \frac{P_{r}}{P_{q}}<\frac{1}{Q-1}, \quad \forall r, q \in \Omega, q \neq r
$$

or [13]

$$
\max _{k \in\{1, \ldots, N\}}\left\{\frac{\left|\bar{H}_{r q}(k)\right|^{2}}{\left|\bar{H}_{q q}(k)\right|^{2}}\right\} \frac{d_{q q}^{\alpha}}{d_{r q}^{\alpha}} \frac{P_{r}}{P_{q}}<\frac{1}{2 Q-3}, \quad \forall r, q \in \Omega, q \neq r
$$

or [14]

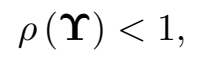

where $\Upsilon$ is defined in (19).

Proof. See Appendix B,

Since the convergence conditions in Corollary 2 depend on the channel realizations $\left\{\bar{H}_{q r}(k)\right\}$ and on the distances $\left\{d_{q r}\right\}$, there is a nonzero probability that they are not satisfied for a given channel realization, drawn from a given probability space. To compare the range of validity of our conditions vs. the conditions available in the literature, we tested them over a set of channel impulse responses generated as vectors composed of $L=6$ i.i.d. complex Gaussian random variables with zero mean and unit variance (multipath Rayleigh fading model). Each user transmits over a set of $N=16$ subcarriers. We consider a multicell cellular network as depicted in Figure 1(a), composed by 7 (regular) hexagonal cells, sharing the same band. Hence, the transmissions from different cells typically interfere with each other. For the simplicity of representation, we assume that in each cell there is only one active link, corresponding to the transmission from the base station (BS) to a mobile terminal (MT) placed at a corner of the cell. According to this geometry, each MT receives a useful signal that is comparable, in average sense, with the interference signal transmitted by the BSs of two adjacent cells. The overall network is modeled as a set of seven wideband interference channels. In Figure 1(b), we plot the

\footnotetext{
${ }^{11}$ Recall that condition (C1) guarantees also the convergence of the more general asynchronous IWFA, as stated in Theorem 1

${ }^{12}$ In [6], the authors derived conditions ([C4) for a game composed by $Q=2$ users and in the absence of spectral mask constraints.
} 


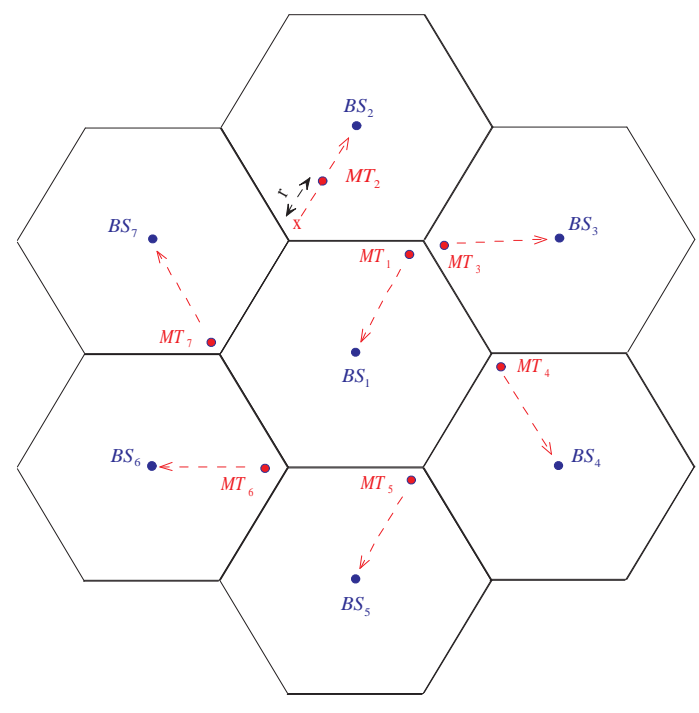

(a) Multicell cellular system

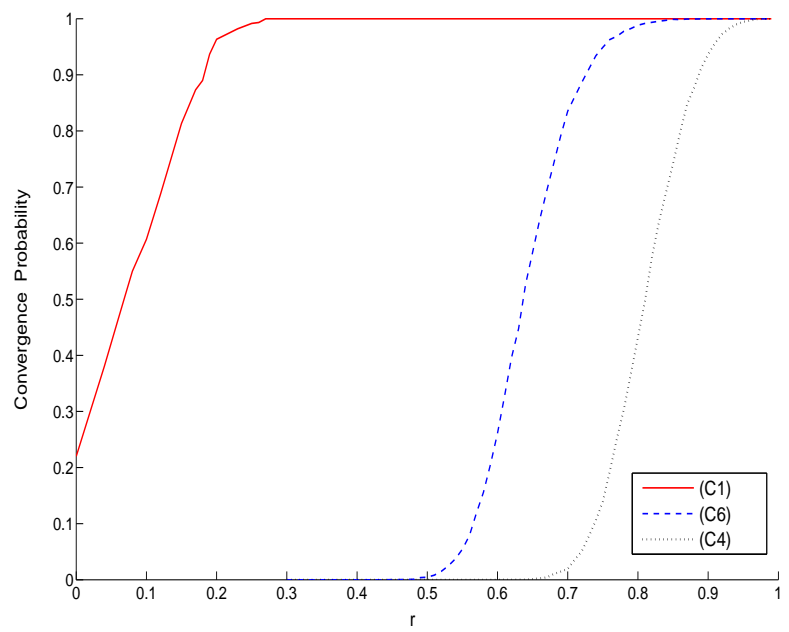

(b) Probability of (C1), (C4) and (C6) versus $r$.

Figure 1: Probability of ([C1), (C4) and (C6) versus $r$ [subplot (b)] for a 7 cell (downlink) cellular system [subplot (a)]; $Q=7, N=16, \gamma=2.5, P_{q}=P_{r}, \Gamma_{q}=1, P_{q} / \sigma_{q}^{2}=7 \mathrm{~dB}, \forall r, q \in \Omega$, and $\boldsymbol{w}=1$.

probability that conditions ( $(\mathrm{C1}),(\underline{\mathrm{C} 4})$ and (C6) are satisfied versus the (normalized) distance $r \in[0,1)$ (see Figure 1(a), , between each MT and his BS (assumed to be equal for all the MT/BS pairs). We tested our conditions considering the set $\mathcal{D}_{q}$, obtained using the algorithm described in [10].

As expected, the probability of guaranteeing convergence increases as each MT approaches his BS (i.e., $r \rightarrow 1$ ). What is worthwhile noticing is that our condition (C1) significantly enlarges (C4) and

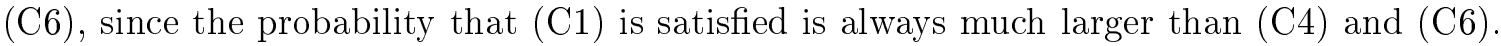

Remark 7 - Sequential versus simultaneous IWFA: Since the simultaneous IWFA in Algorithm 4 is still based on the waterfilling solution (10), it keeps the most appealing features of the sequential IWFA, namely its low-complexity and distributed nature. In addition, it allows all the users to update their optimal power allocation simultaneously. Hence, the simultaneous IWFA is faster than the sequential IWFA, especially if the number of active users in the network is large. A quantitative comparison between the sequential and simultaneous IWFAs, in terms of convergence speed, is given in [11]. In [19], we also provided a closed form expression of the error estimates as a function of the iteration index, obtained by the sequential and simultaneous IWFAs.

\section{Conclusion}

In this paper, we have studied the maximization of the information rates for the Gaussian frequencyselective interference channel, given constraints on the transmit power and the spectral masks on each link. We have formulated the optimization problem as a strategic noncooperative game and we have proposed a novel, totally asynchronous iterative distributed algorithm, named asynchronous IWFA, to reach the Nash equilibria of the game. This algorithm contains as special cases the well-known sequential IWFA and the recently proposed simultaneous IWFA, where the users update their strategies 
sequentially and simultaneously, respectively. The main advantage of the proposed algorithm is that no rigid scheduling in the updates of the users is required: Users are allowed to choose their own strategies whenever they want and some users may even use an outdated version of the measured multiuser interference. This relaxes the coordination requirements among the users significantly. Finally, we have provided the conditions ensuring the global convergence of the asynchronous IWFA to the unique $\mathrm{NE}$ of the game. Interestingly, our convergence conditions do not depend on the specific updating scheduling performed by the users and, hence, they represent a unified set of convergence conditions for all the algorithms that can be seen as special cases of the asynchronous IWFA.

\section{Appendix}

\section{A Proof of Theorems 1 and 2}

We start with some definitions and intermediate results that will be instrumental to prove Theorems 1 and 2 ,

Properties of the waterfilling mapping. For technical reasons, we define the admissible set $\mathscr{P}$ eff $=\mathscr{P}_{1}^{\mathrm{eff}} \times \cdots \times \mathscr{P}_{Q}^{\mathrm{eff}} \subseteq \mathscr{P}$, where

$$
\mathscr{P}_{q}^{\mathrm{eff}} \triangleq\left\{\mathbf{p}_{q} \in \mathscr{P}_{q} \text { with } p_{q}(k)=0 \forall k \notin \mathcal{D}_{q}\right\}
$$

is the subset of $\mathscr{P}_{q}$ containing all the feasible power allocations of user $q$, with zero power over the carriers that user $q$ would never use in any of its waterfilling solutions (10), against any admissible strategy of the others. Observe that the game does not change if we use $\mathscr{P}$ eff instead of the original $\mathscr{P}$. For any given $\left\{\alpha_{q}\right\}_{q \in \Omega}$, with $\alpha_{q} \in[0,1)$, let $\mathbf{T}(\mathbf{p})=\left(\mathbf{T}_{q}(\mathbf{p})\right)_{q \in \Omega}: \mathscr{P}^{\text {eff }} \mapsto \mathscr{P}^{\text {eff }}$ be the mapping defined, for each $q$, as

$$
\mathbf{T}_{q}(\mathbf{p}) \triangleq \alpha_{q} \mathbf{p}_{q}+\left(1-\alpha_{q}\right) \mathrm{WF}_{q}\left(\mathbf{p}_{-q}\right), \quad \mathbf{p} \in \mathscr{P} \mathrm{eff},
$$

where $\mathrm{WF}_{q}\left(\mathbf{p}_{-q}\right): \mathscr{P}_{-q}^{\text {eff }} \mapsto \mathscr{P}_{q}^{\text {eff }}$ is the waterfilling operator defined in (10). Observe that all the Nash equilibria of game $\mathscr{G}$ correspond to the fixed points in $\mathscr{P}$ eff of the mapping $\mathbf{T}$ in (21). Hence, the existence of at least one fixed point for $\mathbf{T}$ is guaranteed by Proposition 1 .

Given $\mathbf{T}$ in (21) and some $\mathbf{w} \triangleq\left[w_{q}, \ldots, w_{Q}\right]^{T}>\mathbf{0}$, let $\|\cdot\|_{2, \text { block }}^{\mathbf{w}}$ denote the (vector) block-maximum norm, defined as [18]

$$
\|\mathbf{T}(\mathbf{p})\|_{2, \text { block }}^{\mathbf{w}} \triangleq \max _{q \in \Omega} \frac{\left\|\mathbf{T}_{q}(\mathbf{p})\right\|_{2}}{w_{q}},
$$

where $\|\cdot\|_{2}$ is the Euclidean norm. Let $\|\cdot\|_{\infty, \text { vec }}^{\mathbf{w}}$ be the vector weighted maximum norm, defined as [27]

$$
\|\mathbf{x}\|_{\infty, \mathrm{vec}}^{\mathbf{w}} \triangleq \max _{q \in \Omega} \frac{\left|x_{q}\right|}{w_{q}}, \quad \mathbf{w}>\mathbf{0}, \quad \mathbf{x} \in \mathbb{R}^{Q}
$$

and let $\|\cdot\|_{\infty, \text { mat }}^{\mathbf{w}}$ denote the matrix norm induced by $\|\cdot\|_{\infty, \text { vec }}^{\mathbf{w}}$, defined as [27]

$$
\|\mathbf{A}\|_{\infty, \text { mat }}^{\mathbf{w}} \triangleq \max _{q} \frac{1}{w_{q}} \sum_{r=1}^{Q}[\mathbf{A}]_{q r} w_{r}, \quad \mathbf{A} \in \mathbb{R}^{Q \times Q} .
$$


Finally, we introduce the matrix $\mathbf{S}_{\alpha}^{\max }$, defined as

$$
\mathbf{S}_{\alpha}^{\max } \triangleq \mathbf{D}_{\alpha}+\left(\mathbf{I}-\mathbf{D}_{\alpha}\right) \mathbf{S}^{\max }, \text { with } \mathbf{D}_{\alpha} \triangleq \operatorname{diag}\left(\alpha_{q} \ldots \alpha_{Q}\right)
$$

where $\mathbf{S}^{\max }$ is defined in (13). The block-contraction property of mapping $\mathbf{T}$ in (21) is given in the following theorem that comes directly from [11, Proposition 2].

Theorem 4 (Contraction property of mapping T) Given $\mathbf{w} \triangleq\left[w_{1}, \ldots, w_{Q}\right]^{T}>\mathbf{0}$ and $\left\{\alpha_{q}\right\}_{q \in \Omega}$, with $\alpha_{q} \in[0,1)$, the mapping $\mathbf{T}$ defined in (21) satisfies

$$
\left\|\mathbf{T}\left(\mathbf{p}^{(1)}\right)-\mathbf{T}\left(\mathbf{p}^{(2)}\right)\right\|_{2, \text { block }}^{\mathbf{w}} \leq\left\|\mathbf{S}_{\alpha}^{\max }\right\|_{\infty, \text { mat }}^{\mathbf{w}}\left\|\mathbf{p}^{(1)}-\mathbf{p}^{(2)}\right\|_{2, \text { block }}^{\mathbf{w}}, \quad \forall \mathbf{p}^{(1)}, \mathbf{p}^{(2)} \in \mathscr{P} \text { eff }
$$

where $\|\cdot\|_{2, \text { block }}^{\mathbf{w}},\|\cdot\|_{\infty, \text { mat }}^{\mathbf{w}}$ and $\mathbf{S}_{\alpha}^{\max }$ are defined in (22), (24) and (25), respectively. If $\left\|\mathbf{S}_{\alpha}^{\max }\right\|_{\infty, \text { mat }}^{\mathbf{w}}<1$, then mapping $\mathbf{T}$ is a block-contraction with modulus $\left\|\mathbf{S}_{\alpha}^{\max }\right\|_{\infty, \text { mat }}^{\mathbf{w}}$.

Asynchronous convergence theorem [18]. Let $\mathcal{X}_{1}, \mathcal{X}_{2}, \ldots, \mathcal{X}_{Q}$ be given sets, and let $\mathcal{X}$ be their Cartesian product, i.e.,

$$
\mathcal{X}=\mathcal{X}_{1} \times \mathcal{X}_{2} \times \ldots \times \mathcal{X}_{Q}
$$

Let $\mathbf{f}_{q}: \mathcal{X} \mapsto \mathcal{X}_{q}$ be a given vector function and let $\mathbf{f}: \mathcal{X} \mapsto \mathcal{X}$ be the mapping from $\mathcal{X}$ to $\mathcal{X}$, defined as $\mathbf{f}(\mathbf{x})=\left(\mathbf{f}_{1}(\mathbf{x}), \ldots, \mathbf{f}_{Q}(\mathbf{x})\right)$, and assumed to admit a fixed point $\mathbf{x}^{\star}=\mathbf{f}\left(\mathbf{x}^{\star}\right)$. Consider the following distributed asynchronous iterative algorithm to reach $\mathbf{x}^{\star}$

$$
\mathbf{x}_{q}^{(n+1)}= \begin{cases}\mathbf{f}_{q}\left(\mathbf{x}_{1}^{\left(\tau_{1}^{q}(n)\right)}, \ldots, \mathbf{x}_{Q}^{\left(\tau_{Q}^{q}(n)\right)}\right), & \text { if } n \in \mathcal{T}_{q}, \quad, \forall q \in \Omega \\ \mathbf{x}_{q}^{(n)}, & \text { otherwise, }\end{cases}
$$

with $0 \leq \tau_{r}^{q}(n) \leq n$ and $\mathcal{T}_{q}$ denoting the set of times $n$ at which $\mathbf{x}_{q}^{(n)}$ is updated and satisfying A1-A3 of Section 3. Assume that:

C.1 (Nesting Condition) There exists a sequence of nonempty sets $\{\mathcal{X}(n)\}$ with

$$
\ldots \subset \mathcal{X}(n+1) \subset \mathcal{X}(n) \subset \ldots \subset \mathcal{X}
$$

satisfying the next two conditions.

\section{C.2 (Synchronous Convergence Condition)}

$$
\mathbf{f}(\mathbf{x}) \in \mathcal{X}(n+1), \quad \forall n, \text { and } \mathbf{x} \in \mathcal{X}(n) .
$$

Furthermore, if $\left\{\mathbf{y}^{(n)}\right\}$ is a sequence such that $\mathbf{y}^{(n)} \in \mathcal{X}(n)$, for every $n$, then every limit point of $\left\{\mathbf{y}^{(n)}\right\}$ is a fixed point of $\mathbf{f}(\cdot)$.

C.3 (Box Condition) For every $n$ there exist sets $\mathcal{X}_{q}(n) \subset \mathcal{X}_{q}$ such that

$$
\mathcal{X}(n)=\mathcal{X}_{1}(n) \times \ldots \times \mathcal{X}_{Q}(n) .
$$


Then we have the following .

Theorem 5 ([18, Proposition 2.1]) If the Synchronous Convergence Condition (30) and the Box Condition (31) are satisfied, and the starting point $\mathbf{x}^{(0)} \triangleq\left(\mathbf{x}_{1}^{(0)}, \ldots, \mathbf{x}_{Q}^{(0)}\right)$ of the algorithm (28) belongs to $\mathcal{X}(0)$, then every limit point of $\left\{\mathbf{x}^{(n)}\right\}$ given by (28) is a fixed point of $\mathbf{f}(\cdot)$.

We are now ready to prove Theorems 1 and 2 through the following two steps:

Step 1. We first show that the asynchronous IWFA in Algorithms 1 and 2 is an instance of the totally asynchronous iterative algorithm in (28). Then, using Theorem 4, we derive sufficient conditions for

\section{C.1-C.3.}

Step 2. Invoking Theorem [5, we complete the proof showing that the asynchronous IWFA converges to the unique NE of $\mathscr{G}$ from any starting point, provided that condition (C1) is satisfied.

Step 1. It is straightforward to see that the asynchronous IWFA coincides with the algorithm given in (28), under the following identifications

$$
\begin{aligned}
& \mathbf{x}_{q} \Leftrightarrow \mathbf{p}_{q}, \quad \mathbf{x}_{q}^{\star} \Leftrightarrow \mathbf{p}_{q}^{\star}, \quad \mathcal{X}_{q} \Leftrightarrow \mathscr{P}_{q}^{\mathrm{eff}}, \quad \forall q \in \Omega, \\
& \mathbf{f}_{q}(\mathbf{x}) \Leftrightarrow \mathbf{T}_{q}(\mathbf{p}), \\
& \mathcal{X} \Leftrightarrow \mathscr{P}^{\mathrm{eff}}=\mathscr{P}_{1}^{\mathrm{eff}} \times \ldots \times \mathscr{P}_{Q}^{\mathrm{eff}},
\end{aligned}
$$

where $\mathscr{P}_{q}^{\text {eff }}$ and $\mathbf{T}_{q}(\mathbf{p})$ are defined in (20) and (21), respectively. Observe that, to study the convergence of the asynchronous IWFA, there is no loss of generality in considering the mapping $\mathbf{T}$ defined in $\mathscr{P}^{\text {eff }} \subset \mathscr{P}$ instead of $\mathscr{P}$, since all the points produced by the algorithm (except possibly the initial point, which does not affect the convergence of the algorithm in the subsequent iterations) as well as the Nash equilibria of the game are confined, by definition, in $\mathscr{P}$ eff.

We consider now conditions C.1-C.3 separately.

C.1 (Nested Condition) Let $\mathbf{p}^{\star}=\left(\mathbf{p}_{1}^{\star}, \ldots, \mathbf{p}_{Q}^{\star}\right) \in \mathscr{P}$ eff be a fixed point of $\mathbf{T}$ in (21) (or, equivalently of $\mathbf{f}_{q}$ in (28) $)$ and $\mathbf{p}^{(0)}=\left(\mathbf{p}_{1}^{(0)}, \ldots, \mathbf{p}_{Q}^{(0)}\right) \in \mathscr{P}^{\text {eff }}$ be any starting point of the asynchronous IWFA. Using the block-maximum norm $\|\cdot\|_{2, \text { block }}^{\mathbf{w}}$ as defined in (22), where $\mathbf{w}=\left[w_{1}, \ldots, w_{q}\right]^{T}$ is any positive vector, we define the set $\mathcal{X}(n)$ in (29) as

$$
\mathcal{X}(n)=\left\{\mathbf{p} \in \mathscr{P}^{\mathrm{eff}}:\left\|\mathbf{p}-\mathbf{p}^{\star}\right\|_{2, \text { block }}^{\mathbf{w}} \leq \beta^{n}\left\|\mathbf{p}^{(0)}-\mathbf{p}^{\star}\right\|_{2, \text { block }}^{\mathbf{w}}\right\} \subset \mathscr{P}^{\mathrm{eff}}
$$

with

$$
\beta=\beta\left(\mathbf{w}, \mathbf{S}_{\alpha}^{\max }\right) \triangleq\left\|\mathbf{S}_{\alpha}^{\max }\right\|_{\infty}^{\mathbf{w}}
$$

and $\mathbf{S}_{\alpha}^{\max }$ defined in (25). It follows from (33) that if

$$
\beta^{n+1}\left\|\mathbf{p}^{(0)}-\mathbf{p}^{\star}\right\|_{2, \text { block }}^{\mathbf{w}}<\beta^{n}\left\|\mathbf{p}^{(0)}-\mathbf{p}^{\star}\right\|_{2, \text { block }}^{\mathbf{w}}, \quad \forall n=0,1, \ldots,
$$

then we obtain the desired result, i.e.,

$$
\mathcal{X}(n+1) \subset \mathcal{X}(n) \subset \mathscr{P}^{\text {eff }}, \quad \forall n=0,1, \ldots
$$


A necessary and sufficient condition for (35) is

$$
\beta<1 \text {. }
$$

We will assume in the following that (36) is satisfied.

C.2 (Synchronous Convergence Condition) Let $\mathbf{p}^{(n)} \in \mathcal{X}(n)$. Then, from (33), it must be that

$$
\left\|\mathbf{p}^{(n)}-\mathbf{p}^{\star}\right\|_{2, \text { block }}^{\mathbf{w}} \leq \beta^{n}\left\|\mathbf{p}^{(0)}-\mathbf{p}^{\star}\right\|_{2, \text { block }}^{\mathbf{w}} .
$$

Let $\mathbf{p}^{(n+1)}=\mathbf{T}\left(\mathbf{p}^{(n)}\right)$. Then, we have

$$
\left\|\mathbf{p}^{(n+1)}-\mathbf{p}^{\star}\right\|_{2, \text { block }}^{\mathbf{w}}=\left\|\mathbf{T}\left(\mathbf{p}^{(n)}\right)-\mathbf{p}^{\star}\right\|_{2, \text { block }}^{\mathbf{w}} \leq \beta\left\|\mathbf{p}^{(n)}-\mathbf{p}^{\star}\right\|_{2, \text { block }}^{\mathbf{w}} \leq \beta^{n+1}\left\|\mathbf{p}^{(0)}-\mathbf{p}^{\star}\right\|_{2, \text { block }}^{\mathbf{w}},
$$

where the first and the second inequalities follow from Theorem 4 (using (26) with $\mathbf{p}^{\star}=\mathbf{T}\left(\mathbf{p}^{\star}\right)$ ) and (37), respectively. Hence, $\mathbf{p}^{(n+1)} \in \mathcal{X}(n+1)$, as required in (30). Furthermore, since

$$
\lim _{n \rightarrow \infty}\left\|\mathbf{p}^{(n)}-\mathbf{p}^{\star}\right\|_{2, \text { block }}^{\mathbf{w}}=0, \text { with } \mathbf{p}^{(n)} \in \mathcal{X}(n), \forall n,
$$

the sequence $\left\{\mathbf{p}^{(n)}\right\}$ generated from $\mathbf{p}^{(0)}$ by the mapping $\mathbf{T}$ using the simultaneous updating scheme in (30) must converge to $\mathbf{p}^{\star}$. Moreover, it follows from (36) and Theorem 4 that the fixed point $\mathbf{p}^{\star}$ of $\mathbf{T}$ is unique (implied from the fact that the mapping $\mathbf{T}$ is a block-contraction [18, Proposition 1.1]).

C.3 (Box Condition) For every $n$, the set $\mathcal{X}(n)$ in (33) can be decomposed as $\mathcal{X}(n)=\mathcal{X}_{1}(n) \times \ldots \times$ $\mathcal{X}_{Q}(n)$, with

$$
\mathcal{X}_{q}(n)=\left\{\mathbf{p}_{q} \in \mathscr{P}_{q}^{\mathrm{eff}}: \frac{\left\|\mathbf{p}_{q}-\mathbf{p}_{q}^{\star}\right\|_{2}}{w_{q}} \leq \beta^{n}\left\|\mathbf{p}^{(0)}-\mathbf{p}^{\star}\right\|_{2, \text { block }}^{\mathbf{w}}\right\} \subset \mathscr{P}_{q}^{\mathrm{eff}}, \quad \forall q \in \Omega .
$$

Step 2. Under (36), Theorem 5 is satisfied if the starting point $\mathbf{p}^{(0)}$ of the asynchronous IWFA is such that $\mathbf{p}^{(0)} \in \mathscr{P}$ eff. The asynchronous IWFA, as given in Algorithm 1 and Algorithm 2, is allowed to start from any arbitrary point $\mathbf{p}^{(0)}$ in $\mathscr{P}$. However, after the first iteration from all the users, the asynchronous IWFA provides a point in $\mathscr{P}$ eff, for any $\mathbf{p}^{(0)} \in \mathscr{P}$. Hence, under ( $\left.\underline{36}\right)$, the asynchronous IWFA satisfies Theorem 5, after the first iteration, which still guarantees that every limit point of the sequence generated by the asynchronous IWFA is a NE of the game $\mathscr{G}$. Since condition (36) is also sufficient for the uniqueness of the NE [recall that, under (36), the mapping $\mathbf{T}$ in (21) is a block-contraction], the asynchronous IWFA must converge to this unique NE.

To complete the proof, we just need to show that (C1) is equivalent to (36). Since in (36) each $\alpha_{q} \in[0,1)$, we have

$$
\beta=\left\|\mathbf{S}_{\alpha}^{\max }\right\|_{\infty}^{\mathbf{w}}<1 \quad \Leftrightarrow \quad\left\|\mathbf{S}^{\max }\right\|_{\infty}^{\mathbf{w}}<1
$$

Since $\mathbf{S}^{\max }$ is a nonnegative matrix, there exists a positive vector $\overline{\mathbf{w}}$ such that $[18$, Corollary 6.1$]$

$$
\left\|\mathbf{S}^{\max }\right\|_{\infty}^{\overline{\mathbf{w}}}<1 \quad \Leftrightarrow \quad \rho\left(\mathbf{S}^{\max }\right)<1
$$


Since the convergence of the asynchronous IWFA is guaranteed under (36), for any given $\mathbf{w}>\mathbf{0}$, we can choose $\mathbf{w}=\overline{\mathbf{w}}$ and use (41).

Conditions (C2)-(C3) in Corollary 1 can be obtained as follows. Using [18, Proposition 6.2e] $\rho\left(\mathbf{S}^{\max }\right) \leq\left\|\mathbf{S}^{\max }\right\|_{\infty}^{\mathbf{w}}, \forall \mathbf{w}>\mathbf{0}$, a sufficient condition for the $\Rightarrow$ direction in (41) is $\left\|\mathbf{S}^{\max }\right\|_{\infty}^{\mathbf{w}}<1$, for

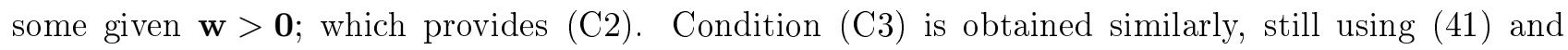
$\rho\left(\mathbf{S}^{\max }\right)=\rho\left(\mathbf{S}^{\max T}\right)$.

\section{B Proof of Corollary 2}

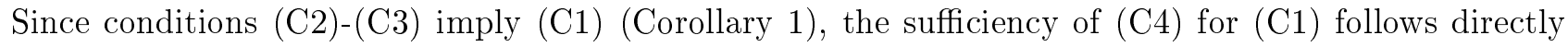
setting, in ( $(\mathrm{C2} 2), P_{q}=P_{r}$ for all $q, r, \mathcal{D}_{q}=\mathcal{D}_{r}=\{1, \ldots, N\}, \mathbf{w}=\mathbf{1}$, and using the following upper bound $\left|\bar{H}_{r q}(k)\right|^{2} /\left|\bar{H}_{q q}(k)\right|^{2} \leq \max _{r \neq q}\left\{\left|\bar{H}_{r q}(k)\right|^{2} /\left|\bar{H}_{q q}(k)\right|^{2}\right\}$. Observe that condition (C5) is stronger than (C4), and thus implies (C1).

We prove now that condition ( $\mathrm{C} 6$ ) is stronger than (C1). To this end, it is sufficient to show that $\rho(\mathbf{\Upsilon})<1 \Leftrightarrow \rho\left(\overline{\mathbf{S}}^{\max }\right)<1$, where $\overline{\mathbf{S}}^{\max }$ is defined after (19), since $\mathbf{S}^{\max } \leq \overline{\mathbf{S}}^{\max }$ leads to $\rho\left(\mathbf{S}^{\max }\right) \leq$ $\rho\left(\overline{\mathbf{S}}^{\max }\right)<1[28$, Corollary 2.2.22] 13 We first introduce the following intermediate definition and result $[28]$.

Definition 2 A matrix $\mathbf{A} \in \mathbb{R}^{n \times n}$ is said to be a $\mathbf{Z}$-matrix if its off-diagonal entries are all non-positive. A matrix $\mathbf{A} \in \mathbb{R}^{n \times n}$ is said to be a $\mathbf{P}$-matrix if all its principal minors are positive. $A \mathbf{Z}$-matrix that is also $\mathbf{P}$ is called a $\mathbf{K}$-matrix.

Lemma 1 ([28, Lemma 5.3.14]) Let $\mathbf{A} \in \mathbb{R}^{n \times n}$ be a $\mathbf{K}$-matrix and $\mathbf{B}$ a nonnegative matrix. Then $\rho\left(\mathbf{A}^{-1} \mathbf{B}\right)<1$ if and only if $\mathbf{A}-\mathbf{B}$ is a $\mathbf{K}$-matrix.

According to Definition [2, $\mathbf{I}-\overline{\mathbf{S}}_{\text {low }}^{\max }$ is a $\mathbf{Z}$-matrix. Since all principal minors of $\mathbf{I}-\overline{\mathbf{S}}_{\text {low }}^{\text {max }}$ are equal to one (recall that $\mathbf{I}-\overline{\mathbf{S}}_{\text {low }}^{\max }$ is a lower triangular matrix with all ones on the main diagonal), $\mathbf{I}-\overline{\mathbf{S}}_{\text {low }}^{\max }$ is also a $\mathbf{P}$-matrix, and thus a K-matrix. Invoking Lemma 1 we obtain the following chain of equivalences

$$
\rho(\mathbf{\Upsilon})<1 \quad \Leftrightarrow \quad \mathbf{I}-\overline{\mathbf{S}}^{\max } \text { is a } \mathbf{K} \text {-matrix } \Leftrightarrow \rho\left(\overline{\mathbf{S}}^{\max }\right)<1
$$

where the first and the second equivalence follows from Lemma 1 using the correspondences $\mathbf{A}=$ $\mathbf{I}-\overline{\mathbf{S}}_{\text {low }}^{\max }, \mathbf{B}=\overline{\mathbf{S}}_{\text {upp }}^{\max }, \mathbf{I}-\overline{\mathbf{S}}_{\text {low }}^{\max }-\overline{\mathbf{S}}_{\text {upp }}^{\max }=\mathbf{I}-\overline{\mathbf{S}}^{\text {max }}$ and $\mathbf{A}=\mathbf{I}, \mathbf{B}=\overline{\mathbf{S}}^{\text {max }}$, respectively. It follows from (42) that $\rho(\mathbf{\Upsilon})<1 \Leftrightarrow \rho\left(\overline{\mathbf{S}}^{\max }\right)<1 \Rightarrow \rho\left(\mathbf{S}^{\max }\right)<1$; which completes the proof.

\section{References}

[1] T. S. Han, and K. Kobayashi, "A new achievable rate region for the interference channel", IEEE Trans. on Inform. Theory, vol. 27, pp. 49-60, Jan. 1981.

\footnotetext{
${ }^{13}$ The inequality $\mathbf{S}^{\max } \leq \overline{\mathbf{S}}^{\max }$ has to be intended componentwise.
} 
[2] E. C. van der Meulen, "Some Reflections on the Interference Channel", Boston, MA, Kluwer, pp. 409-421, 1994.

[3] S. Haykin, "Cognitive Radio: Brain-Empowered Wireless Communications", in IEEE Jour. on Selected Areas in Comm., vol. 23, no. 2, pp. 201-220, February 2005.

[4] R. Cendrillon, W. Yu, M. Moonen, J. Verlinder, and T. Bostoen, "Optimal Multi-User Spectrum Managment for Digital Subscriber Lines", in IEEE Trans. on Commun., Vol. 54, No. 5, pp. 922 - 933, May 2006.

[5] W. Yu, and Raymond Lui, "Dual Methods for Nonconvex Spectrum Optimization of Multicarrier Systems", IEEE Trans. on Commun., vol. 54, no. 7, pp. 1310-1322, July 2006.

[6] W. Yu, G. Ginis, and J. M. Cioffi, "Distributed multiuser power control for digital subscriber lines", IEEE J. Select. Areas Commun., vol. 20, pp. 1105-1115, June 2002.

[7] S. T. Chung, S. J. Kim, J. Lee, and J. M. Cioffi, "A game-theoretic approach to power allocation in frequency-selective Gaussian interference channels", in Proc. 2003 IEEE Int. Symp. on Inform. Theory (ISIT 2003), p. 316, June 2003.

[8] G. Scutari, S.Barbarossa, and D.Ludovici, "Cooperation Diversity in Multihop Wireless Networks Using Opportunistic Driven Multiple Access,"in Proc. of the 2003 IEEE Workshop on Sig. Proc. Advances in Wireless Comm., (SPAWC-2003), pp. 170-174, June 2003.

[9] G. Scutari, D. P. Palomar, and S. Barbarossa, "Simultaneous Iterative Water-Filling for Gaussian Frequency-Selective Interference Channels", in Proc. 2006 IEEE Int. Symp. on Inform. Theory (ISIT 2006), July 2006 .

[10] G. Scutari, D. P. Palomar, and S. Barbarossa, "Optimal Linear Precoding Strategies for Wideband Non-Cooperative Systems based on Game Theory-Part I: Nash Equilibria," to appear on IEEE Trans. on Signal Processing, 2008. Available at http://arxiv.org/abs/0707.0568.

[11] G. Scutari, D. P. Palomar, and S. Barbarossa, "Optimal Linear Precoding Strategies for Wideband Non-Cooperative Systems based on Game Theory-Part II: Algorithms," to appear on IEEE Trans. on Signal Processing, 2008. Available at http://arxiv.org/abs/0707.0871v1.

[12] G. Scutari, Competition and Cooperation in Wireless Communication Networks , PhD. Dissertation, INFOCOM Dep., University of Rome, "La Sapienza", November 2004.

[13] N. Yamashitay and Z. Q. Luo, "A Nonlinear Complementarity Approach to Multiuser Power Control for Digital Subscriber Lines", Optimization Methods and Software, vol. 19, pp. 633-652, 2004 .

[14] Z.-Q. Luo and J.-S. Pang, "Analysis of Iterative Waterfilling Algorithm for Multiuser Power Control in Digital Subscriber Lines," in EURASIP J. on Applied Sig. Proc., Article ID 24012, pp. 1-10, April 2006.

[15] R. Etkin, A. Parekh, D. Tse, "Spectrum sharing for unlicensed bands", in Proc. of the Allerton Conference on Communication, Control, and Computing, Monticello, IL, Sept. 28-30, 2005.

[16] S. Boyd and L. Vandenberghe, Convex optimization, Cambridge University Press, 2003.

[17] J. Nash, "Equilibrium Points in $n$ person Game", in Proc. National Academy of Science , vol. 36, pp. $48-49,1950$.

[18] D. P Bertsekas and J.N. Tsitsiklis, Parallel and Distributed Computation: Numerical Methods , Athena Scientific, 2nd Ed., 1989. 
[19] G. Scutari, D. P. Palomar, and S. Barbarossa, "Asynchronous Iterative Waterfilling for Gaussian Frequency-Selective Interference Channels: A Unified Framework," in Proc. Information Theory and Applications (ITA) Workshop, San Diego, CA, USA, Jan. 29 - Feb. 2, 2007.

[20] G. Scutari, D. P. Palomar, and Sergio Barbarossa, "Distributed Totally Asynchronous Iterative Waterfilling for Wideband Interference Channel with Time/Frequency Offset," in Proc. of the IEEE Int. Conf. on Acoustics, Speech, and Signal Processing (ICASSP) , Honolulu, Hawaii, USA, April 15-20, 2007.

[21] T. M. Cover and J. A. Thomas, Elements of Information Theory, John Wiley and Sons, 1991.

[22] D. Tse and P. Viswanath Fundamentals of Wireless Communication, Cambridge University Press, 2005.

[23] M. J. Osborne and A. Rubinstein, A Course in Game Theory, MIT Press, 1994.

[24] J. P. Aubin, Mathematical Method for Game and Economic Theory, Elsevier, Amsterdam, 1980.

[25] J. G. David Forney and M. V. Eyuboglu, "Combined equalization and coding using precoding", IEEE Commun. Mag., vol. 29, no. 12, pp. 25-34, Dec. 1991.

[26] A. J. Goldsmith and S.-G. Chua, "Variable-rate variable-power MQAM for fading channels", IEEE Trans. on Commun., vol. 45, no. 10, pp. 1218-1230, Oct. 1997.

[27] R. Horn, and C. R. Johnson, Matrix Analysis , Cambridge University Press, 1985.

[28] R.W. Cottle, J.-S. Pang, and R.E. Stone, The Linear Complementarity Problem , Academic Press (Cambridge 1992). 